\title{
An energy dense, high saturated fat, low-fibre dietary pattern is longitudinally associated with CVD risk factors in a severely obese cohort
}

\author{
D. J. Johns ${ }^{1}$, G. L. Ambrosini ${ }^{1}$, S. A. Jebb ${ }^{1}$, L. Sjöström ${ }^{2}$, L. M. S. Carlsson ${ }^{2}$ and A. K. Lindroos ${ }^{3}$ \\ ${ }^{1}$ MRC Human Nutrition Research, Cambridge, UK, ${ }^{2}$ Institute of Medicine, University of Gothenburg, Sweden and \\ ${ }^{3}$ The National Food Administration, Box 622, SE - 75126 Uppsala, Sweden
}

A dietary pattern approach is useful in the study of diet and health outcomes as it accounts for the potentially cumulative effects of the whole diet. We investigated the longitudinal associations between a dietary pattern (DP) and anthropometric and biochemical metabolic disease risk factors measured repeatedly over a 10-year period in a population with severe obesity and at high risk of CVD.

Dietary intake among 2037 severely obese individuals (BMI $41 \pm 4 \mathrm{~kg} / \mathrm{m}^{2}$ ) from the control group of the Swedish Obese Subjects (SOS) study was assessed nine times over 10-years using a validated dietary questionnaire. At registration into the study a DP was derived using reduced rank regression (RRR), based on intakes of thirty-nine food groups, with dietary energy density, saturated fat and fibre density as response variables. A score for this DP was calculated at each follow-up using confirmatory RRR. Waist, BMI and weight were measured at each follow up $(0,1 / 2,1,2,3,4,6,8$ and 10 years). Fasting total cholesterol, HDL, glucose and insulin were measured at registration, 0,2 and 10 years. Mixed models were used to examine longitudinal associations between dietary pattern scores and these cardiovascular risk factors. Anthropometric models were adjusted for sex, age, smoking and physical activity. Blood lipids, glucose and insulin models were adjusted for sex, age, smoking, physical activity, BMI and relevant medication use.

The DP explained 71.2, 31 and $60.4 \%$ of the variation in dietary energy density (DED), \% energy from saturated fat (SF) and fibre density (FD), respectively. It was positively weighted towards SF $(w=0.44)$ and DED $(w=0.66)$ and inversely weighted to FD $(w=-0.61)$. The DP was characterised by high intakes of chocolate, full-fat-spreads and low-fibre bread, and low intakes of fruits and vegetables. A one SD unit higher dietary pattern score at registration was associated with an increase on average, of 0.68 (SD 0.28$) \mathrm{mU} / 1$ insulin $(P<0.0005), 0.044$ (SD 0.012) mmol/l total cholesterol $(P<0.005), 1.87$ (SD 0.31$) \mathrm{kg}$ weight $(P<0.0001), 1.19$ (SD 0.34$) \mathrm{cm}$ waist circumference $(P<0.0001)$ and $0.66(\mathrm{SD} 0.11) \mathrm{kg} / \mathrm{m}^{2}$ BMI $(P<0.0001)$ at follow up. There were no significant associations between the DP and HDL or blood glucose for any of the follow-up examinations.

Repeated measures of an energy-dense, high saturated fat, low-fibre DP are longitudinally associated with fasting insulin, total cholesterol, weight, waist circumference and BMI in this severely obese population. 